\title{
Slow Relaxation and Diffusion in Holographic Quantum Critical Phases
}

\author{
Richard A. Davison" \\ Department of Physics, Harvard University, Cambridge, Massachusetts 02138, USA \\ Department of Applied Mathematics and Theoretical Physics, University of Cambridge, Cambridge CB3 OWA, United Kingdom \\ Simon A. Gentle \\ Institute for Theoretical Physics, Utrecht University, 3508TD Utrecht, Netherlands \\ and Instituut-Lorentz for Theoretical Physics, Leiden University, 2333CA Leiden, Netherlands \\ Blaise Goutéraux $\oplus^{\ddagger}$ \\ Nordita, KTH Royal Institute of Technology and Stockholm University, Roslagstullsbacken 23, \\ SE-106 91 Stockholm, Sweden
}

(Received 16 January 2019; revised manuscript received 12 April 2019; published 4 October 2019; corrected 23 June 2021)

\begin{abstract}
The dissipative dynamics of strongly interacting systems are often characterized by the timescale set by the inverse temperature $\tau_{P} \sim \hbar /\left(k_{B} T\right)$. We show that near a class of strongly interacting quantum critical points that arise in the infrared limit of translationally invariant holographic theories, there is a collective excitation (a quasinormal mode of the dual black hole spacetime) whose lifetime $\tau_{\text {eq }}$ is parametrically longer than $\tau_{P}: \tau_{\mathrm{eq}} \gg T^{-1}$. The lifetime is enhanced due to its dependence on a dangerously irrelevant coupling that breaks the particle-hole symmetry and the invariance under Lorentz boosts of the quantum critical point. The thermal diffusivity (in units of the butterfly velocity) is anomalously large near the quantum critical point and is governed by $\tau_{\text {eq }}$ rather than $\tau_{P}$. We conjecture that there exists a long-lived, propagating collective mode with velocity $v_{s}$, and in this case the relation $D=v_{s}^{2} \tau_{\text {eq }}$ holds exactly in the limit $T \tau_{\text {eq }} \gg 1$. While scale invariance is broken, a generalized scaling theory still holds provided that the dependence of observables on the dangerously irrelevant coupling is incorporated. Our work further underlines the connection between dangerously irrelevant deformations and slow equilibration.
\end{abstract}

DOI: 10.1103/PhysRevLett.123.141601

In many-body quantum systems with strong interactions, the characteristic timescales relevant for a variety of dynamical processes are short, and are set by the inverse temperature $\tau_{P}=\hbar /\left(k_{B} T\right)$ [1]. For example, $\tau_{P}$ has been shown to control the onset of hydrodynamics in holographic plasmas, the postquench equilibration of the Sachdev-Ye-Kitaev model, as well as the Lyapunov exponent characterizing the growth rate of chaos in both of the aforementioned kinds of theories [2-7]. Transport measurements in the strange metallic phase of high- $T_{c}$ superconductors (HTSCs) $[8,9]$ further support the conjecture that $\tau_{P}$ fundamentally bounds the dynamics of strongly correlated phases [10-14].

Indeed, in the vicinity of a quantum critical point (QCP), $T$ is the only energy scale and so the importance of $\tau_{P}$ is manifest [15]. However, there are circumstances in which nonuniversal effects are important and lead to dynamics

Published by the American Physical Society under the terms of the Creative Commons Attribution 4.0 International license. Further distribution of this work must maintain attribution to the author(s) and the published article's title, journal citation, and DOI. Funded by SCOAP . that survive on timescales much longer than $\tau_{P}$. The most familiar example is near a QCP where translational symmetry is broken by an irrelevant coupling $g$ [16-20], leading to the slow relaxation of momentum and a parametrically small resistivity. More generally, whenever the dynamics near a QCP is sensitive to a dangerously irrelevant coupling, $\tau_{P}$ is no longer privileged since the irrelevant coupling provides an additional energy scale [21]. In such situations, it is not obvious what the relevant timescales for dynamical processes are.

We study a class of strongly interacting, $(d+1)$ dimensional, translationally invariant systems whose infrared (IR) physics are governed by hyperscaling violating QCPs with dynamical exponent $z=1$. The particle-hole symmetry and the invariance under Lorentz boosts of the $T=0$ IR QCP are broken by an irrelevant deformation with coupling $g \propto \rho$ the density of the state. We show that in these systems the incoherent current (i.e., the part of the electric current without momentum drag [22]) acquires a long lifetime $\tau_{\text {eq }}$

$$
\tau_{\text {eq }} \sim \tau_{P}\left(\frac{T^{\Delta_{g}}}{g}\right)^{2},
$$


which is parametrically longer than $\tau_{P}, \tau_{\text {eq }} \gg T^{-1}$, and is controlled by the dimension of the coupling $\Delta_{g}<0$. While we expect typical excitations to have a lifetime $\tau_{P}$, it is only after a time $\tau_{\text {eq }}$ that local equilibration will be achieved and the expected hydrodynamic behavior will take over. The slowly relaxing mode produces a narrow peak in the optical conductivity

$$
\sigma(\omega)=\frac{\rho^{2}}{s T+\mu \rho} \frac{i}{\omega}+\frac{\sigma_{o}}{\left(1-i \omega \tau_{\mathrm{eq}}\right)},
$$

where $\sigma_{o}$ and $\tau_{\mathrm{eq}}$ are given by Eq. (13), $\rho$ is the charge density, $\mu$ the chemical potential, and $s$ the entropy density. We expect that adding slow momentum relaxation to our theories (as in, e.g., Refs. [23-25]) will broaden the divergent $\omega \rightarrow 0$ contribution to the conductivity, Eq. (2), into a Drude-like peak. The interplay between multiple irrelevant deformations can be subtle but important for transport near QCPs [19,20,26-28].

We study these systems using gauge-gravity duality, where the IR QCP is captured by a spacetime metric that is conformal to $\mathrm{AdS}_{d+2}$ and is a solution of Einstein-Dilaton theories with an exponential potential $[29,30]$. It is important to note that our models do not capture competing phases on either side of a QCP, only the dynamics of the quantum critical region itself. The irrelevant deformation is realized by a Maxwell field, with exponential coupling to the dilaton, that backreacts on this spacetime and drives a renormalization group ( $R G)$ flow to a nonzero density ultraviolet (UV) fixed point. In gravitational language, we show that certain charged, translationally invariant, asymptotically $\mathrm{AdS}_{d+2}$ black branes have quasinormal modes with parametrically long lifetimes $\sim \tau_{\text {eq }}$.

Near the QCP, we furthermore show that $\tau_{\text {eq }}$ is the timescale relevant for transport processes that do not involve the dragging of momentum. Specifically, at times $t \gtrsim \tau_{\text {eq }}$, these processes are diffusive. Near the QCP, they are characterized by a single diffusivity $D_{T}$ (the thermal diffusivity) where

$$
D_{T}=\frac{2}{d+1-\theta} v_{B}^{2} \tau_{\mathrm{eq}},
$$

$\theta$ is a universal number quantifying the violation of hyperscaling at the QCP, and $v_{B}$ is the "butterfly" velocity at which quantum chaos spreads. The large value of $D_{T}$ resulting from its sensitivity to irrelevant deformations was established in Ref. [25], and was in potential tension with the upper bounds on diffusivities proposed to ensure the causality of diffusive hydrodynamics [31,32]. The result [Eq. (3)] elegantly resolves this potential tension: at precisely the timescales at which causality appears to be violated, the diffusive hydrodynamic description breaks down due to the existence of the slowly relaxing mode. This is a consequence of the nontrivial fact that both $D_{T}$ and $\tau_{\text {eq }}$ are governed by the same irrelevant deformation of the QCP.

A number of recent works have established relations similar to Eq. (3) between thermal diffusivities and the spreading of quantum chaos [12,25,33-40]. In holographic theories, these have always been of the form $D_{T} \sim v_{B}^{2} \tau_{P}$. Our result, Eq. (3), lends further support to the claim that in general the timescale appearing in this relation should be $\tau_{\text {eq }}$, and not $\tau_{P}$ or the Lyapunov time $\tau_{L}$ (which governs the growth rate of quantum chaos) [31,32]. These timescales could not be distinguished in previous examples, which had $\tau_{\text {eq }} \sim \tau_{L} \sim \tau_{P}$ [41]. Our results are also nontrivially consistent with the quantum hydrodynamic theory for maximally chaotic systems proposed in Ref. [40] and explored in Ref. [42]. The result $D_{T} \sim v_{B}^{2} \tau_{P}$ follows from this theory provided that diffusive hydrodynamics applies at timescales $t \sim \tau_{P}$. Assuming the validity of this theory for the holographic QCPs we study, the parametrically large value of $D_{T}$ therefore implies that hydrodynamics must break down at timescales $t \sim \tau_{\text {eq }} \gg \tau_{P}$, as we explicitly show.

Another consequence of the additional energy scale $g$ in the IR theory is the violation of naive $\omega / T$ scaling in response functions near the QCP. We close by illustrating this explicitly, and by showing that if one carefully takes into account the $g$ dependence of the critical contribution to the conductivity, a generalized scaling theory [23,43-45], which has been applied to dc transport in cuprate strange metals [46], continues to hold. Nontrivial scaling theories near QCPs are attractive from a phenomenological point of view: we know that if the strange metallic phase of high $T_{c}$ superconductors does originate from a QCP, then it cannot be governed by a simple, scale invariant theory, as such a theory is inconsistent with the observed $T$-linear resistivity [47].

In the remainder of this Letter, we describe our setup and outline the calculations leading to the results mentioned above. We have also found analogous results to Eqs. (1) and (3) in a closely related class of systems that are particle-hole symmetric and flow to QCPs with dangerously irrelevant translational symmetry-breaking deformations. The results for these systems, along with a number of technical details, are presented in Ref. [45].

Holographic quantum criticality.-In holographic theories, quantum critical states with dynamical exponent $z=1$ can be described by the $(d+2)$-dimensional EinsteinDilaton action [29]

$$
S_{\text {crit }}=\int d^{d+2} x \sqrt{-g}\left(R-\frac{1}{2}(\partial \phi)^{2}-V_{0} e^{-\delta \phi}\right) .
$$

We study theories where Eq. (4) is the effective action capturing the low temperature dynamics far from the boundary of an asymptotically $\mathrm{AdS}_{d+2}$ spacetime. By identifying the extra spatial dimension with the energy scale of a dual quantum field theory, Eq. (4) describes the 
IR dynamics that arise at the endpoint of a $R G$ flow generated by deforming an UV CFT. The details of the RG flow will determine the constants $V_{0}$ and $\delta$, but are otherwise not important for our analysis.

The quantum critical dynamics are captured by the following classical solutions of the action $[29,30]$, in which the metric transforms covariantly under the $z=1$ rescaling $(t, \vec{x}) \mapsto \lambda(t, \vec{x})$

$$
\begin{aligned}
d s^{2} & =\left(\frac{u}{L}\right)^{2(\theta / d)-2}\left(-L_{t}^{2} d t^{2}+\tilde{L}^{2} d u^{2}+L_{x}^{2} d \vec{x}^{2}\right), \\
\tilde{L}^{2} & =\frac{(d+1-\theta)(d-\theta)}{-V_{0}}, \\
\phi & =\kappa \ln \left(\frac{u}{L}\right), \quad \kappa^{2}=\frac{2}{d} \theta(\theta-d), \quad \kappa \delta=2 \frac{\theta}{d} .
\end{aligned}
$$

$u$ is the radial coordinate in the IR region of the spacetime $u \gg L$. The running dilaton leads to violation of hyperscaling, parametrized by $\theta<0$ (consistent with the null energy condition). At small temperatures, the entropy density $s \sim T^{d-\theta}[48]$ and so the critical state can be thought of as a "CFT" in $(d-\theta)$ spatial dimensions $[49,50] . L_{t}, L_{x}$, and $L$ are functions of the deformations of the UV fixed point, and depend on the details of the RG flow. These length scales typically depend smoothly on the scalar source at the boundary (the deformation of the UV CFT) as it is varied over a continuous range of real values. Each such value allows us to represent a distinct QCP. From a gravitational perspective these are perhaps better thought of as quantum critical lines [14,51-53].

The RG flow away from the IR critical point produces corrections to the solution Eq. (5) in inverse powers of $u / L$. For our purposes, the most important correction comes from the Maxwell action

$$
\Delta S_{\mathrm{irr}}=\int d^{d+2} x \sqrt{-g} \frac{Z_{0}}{4} e^{\gamma \phi} F_{\mu \nu} F^{\mu \nu},
$$

where the constants $Z_{0}$ and $\gamma$ depend on the details of the flow to the UV fixed point. $\gamma$ encodes the dimension of an irrelevant deformation, as we will shortly illustrate.

Solving the Maxwell equations in the spacetime [Eq. (5)] gives the profile of the gauge field at leading order in large $u / L$

$A=A_{0}\left(\frac{u}{L}\right)^{\zeta-1} L_{t} d t, \quad \zeta=d-\kappa \gamma-(d-2) \frac{\theta}{d}$.

The density $\rho=-Z C^{d / 2} A^{\prime} / \sqrt{B D} \propto A_{0}$ at $T=0$, so while the gauge field does not backreact on the metric at the QCP, particle-hole symmetry is broken at all temperatures. $A_{0}$ is the bulk quantity corresponding to the dangerously irrelevant coupling $g$ we referred to in the introduction. Indeed, the gauge field sources corrections to the solution Eq. (5) for the metric and dilaton, which at leading order in $A_{0}$ are $\sim 1+\# A_{0}^{2} u^{2 \Delta_{A_{0}}}$ with $\Delta_{A_{0}}=(d-\theta+\zeta) / 2$. This is an irrelevant deformation if $\Delta_{A_{0}}<0$ (so the corrections vanish as $u / L \rightarrow \infty$ ), which we demand from now on. Treating $u$ as an energy scale in the usual way indeed determines the dimension of the irrelevant coupling $A_{0}$ to be $\Delta_{A_{0}}$ and that of the corresponding irrelevant operator to be $\Delta_{\text {irr }}=$ $d+1-\theta-\Delta_{A_{0}}$ [45]. Therefore, $\Delta_{g}=\Delta_{A_{0}}$ in Eq. (1).

Charge response near the QCP.- In order to compute the optical conductivity, we embed the preceding IR theory into a complete holographic RG flow described by the action

$S=\int d^{d+2} x \sqrt{-g}\left(R-\frac{1}{2}(\partial \phi)^{2}-\frac{Z(\phi)}{4} F^{2}-V(\phi)\right)$,

where $V(\phi)$ and $Z(\phi)$ are chosen to reproduce the IR action $(4)+(6)$ as $\phi \rightarrow \infty$. The states we are interested in are captured by the ansatz for the metric $d s^{2}=-D(r) d t^{2}+$ $B(r) d r^{2}+C(r) d \vec{x}^{2}$, gauge field $A=A(r) d t$, and scalar $\phi=\phi(r) . r$ is a radial coordinate that goes to zero at the boundary, where the metric is asymptotically AdS and $A(0)=\mu \neq 0$ defines the chemical potential of the state. We are interested in thermal states, and so we assume there is a regular black brane horizon at $r=r_{h}>0$, where $\quad D\left(r \rightarrow r_{h}\right)=4 \pi T\left(r_{h}-r\right)+\cdots, \quad B\left(r \rightarrow r_{h}\right)=$ $1 /\left(4 \pi T\left(r_{h}-r\right)\right)+\cdots, C\left(r \rightarrow r_{h}\right)=C_{h}+\cdots, \phi\left(r \rightarrow r_{h}\right)=$ $\phi_{h}+\cdots, A\left(r \rightarrow r_{h}\right)=A_{h}\left(r_{h}-r\right)+\cdots$ The charge and entropy densities are given by the $r$-independent expressions $\rho=-Z C^{d / 2} A^{\prime} / \sqrt{B D}=Z_{h} A_{h} C_{h}^{d / 2}$ and $s=-[\rho A-$ $\left.C^{1+d / 2}(D / C)^{\prime} / \sqrt{B D}\right] / T=4 \pi C_{h}^{d / 2}$, where $Z_{h} \equiv Z\left(\phi\left(r_{h}\right)\right)$. We are mainly interested in the low $T$ solutions that reduce to Eq. (5) in the IR as $T \rightarrow 0$.

The optical conductivity is given by

$$
\sigma(\omega) \equiv-\frac{i}{\omega} \lim _{r \rightarrow 0}\left(r^{2-d} \frac{a_{x}^{\prime}(r)}{a_{x}(r)}\right),
$$

where $a_{x}$ is the ingoing linear perturbation of the spatial component of the gauge field and obeys the equation [22]

$$
\frac{d}{d r}\left[F G \tilde{a}_{x}^{\prime}\right]+\omega^{2} \frac{G}{F} \tilde{a}_{x}=0,
$$

with $\tilde{a}_{x} \equiv a_{x} /(s T+\rho A), \quad F \equiv \sqrt{D / B}, G \equiv Z C^{(d / 2)-1} \times$ $(s T+\rho A)^{2}$.

To calculate the low frequency optical conductivity, we use the usual perturbative ansatz [54]

$\tilde{a}_{x}=c\left(\frac{r_{h}-r}{r_{h}}\right)^{-i \omega / 4 \pi T}\left(1+\sum_{n=1}^{\infty}\left(\frac{i \omega}{4 \pi T}\right)^{n} \mathcal{A}_{n}(r)\right)$,

where $\mathcal{A}_{n}\left(r_{h}\right)=0$. Substituting this into Eq. (10) and solving at $O(\omega)$ gives

$$
\mathcal{A}_{1}(r)=\int_{r_{h}}^{r} d \tilde{r}\left[\alpha \frac{C}{D} \frac{d}{d \tilde{r}}\left(\frac{1}{s T+\rho A}\right)-\frac{1}{r_{h}-\tilde{r}}\right],
$$


where $\alpha=s^{3} T^{3} Z_{h} \rho^{-2}(s / 4 \pi)^{-2 / d}$. This results in an optical conductivity [Eq. (2)] where

$\sigma_{o}=\frac{s^{2} T^{2} Z_{h}}{(s T+\rho \mu)^{2}}\left(\frac{s}{4 \pi}\right)^{1-2 / d}, \quad \tau_{\mathrm{eq}}=-\frac{\mathcal{A}_{1}(0)}{4 \pi T}$.

The first term in the optical conductivity [Eq. (2)] is the usual small $\omega$ divergence due to momentum conservation, while the second term arises from charge-carrying processes in which no momentum flows [22]. The pole in the second term at $\omega=-i \tau_{\mathrm{eq}}^{-1}$ indicates the existence of a collective excitation with lifetime $\tau_{\text {eq }}$. The result [Eq. (13)] for $\tau_{\text {eq }}$ can only be trusted if $\tau_{\text {eq }} T \gg 1$, as the perturbative expansion is reliable for $\omega \ll T$.

For a low $T$ state that is sufficiently close to the QCP described by Eqs. (5) and (7), we will now verify that indeed $\tau_{\text {eq }}$ is parametrically longer than $T^{-1}$. The deep IR geometry of such a state will have an event horizon at a large value of $u=u_{h}$, but will still be described by Eqs. (5) and (7) over the range $u_{\mathrm{IR}}>u>u_{\mathrm{UV}}$, with $u_{h} \gg u_{\mathrm{IR}}$ and $u_{\mathrm{UV}} \gg L$. Integrating over this part of the spacetime yields a contribution to $\tau_{\text {eq }}$ that is independent of the cutoffs [45]:

$$
\tau_{\text {eq }}=\frac{\tilde{L}(d+1-\theta)}{L_{t} Z_{0}(1-\zeta)^{2}} \frac{1}{A_{0}^{2}}\left(\frac{u_{h}}{L}\right)^{1-2 \Delta_{A_{0}}} \sim \frac{1}{T} \frac{T^{2 \Delta_{A_{0}}}}{A_{0}^{2}} .
$$

Recalling that $\Delta_{A_{0}}<0$, this contribution to $\tau_{\mathrm{eq}}$ is parametrically larger than $T^{-1}$ and should dominate the full integral in the limit $T \rightarrow 0$. It is manifest that the irrelevant deformation sourced by $A_{0}$ is responsible for the slow relaxation of the mode, and indeed $\tau_{\mathrm{eq}}$ is of the form given in Eq. (1) with $g \sim A_{0}$ and $\Delta_{g}=\Delta_{A_{0}}$.

Counterparts of the QCPs [Eq. (5)] with $z \neq 1$ are well known $[29,30,48]$. For these solutions, the deformation parametrized by $A_{0}$ is marginal $\left(\Delta_{A_{0}}=0\right)$, and the integral for $\tau_{\text {eq }}$ is no longer dominated by the IR spacetime. In these cases we expect $\tau_{\mathrm{eq}} \sim 1 / T$, as has been observed numerically in a variety of holographic theories [55-61].

Diffusivity and hydrodynamics.-As mentioned above, there are two distinct contributions to the small $\omega$ optical conductivity [Eq. (2)]. The divergence at $\omega \rightarrow 0$ is due to current $(J)$ flow that drags (conserved) momentum $(P)$, while the remainder is due to current flow that does not. The latter processes can be conveniently isolated by examining the dynamics of the "incoherent" current $J_{\text {inc }} \equiv \chi_{P P} J-\chi_{J P} P$, where $\chi$ denote static susceptibilities [22]. We will concentrate on $J_{\text {inc }}$ : its small $\omega$ conductivity $\sigma_{\text {inc }}(\omega)$ is proportional to the second term of Eq. (2), and is sensitive to the slowly relaxing mode [62].

Over sufficiently long timescales, we expect relativistic hydrodynamics to govern the system and thus the conductivity of $J_{\text {inc }}$ to be $\omega$ independent [22]. From Eq. (2), it is apparent that this is the case at times $t \gg \tau_{\text {eq. }}$. In this regime, long wavelength perturbations of $J_{\text {inc }}$ and its associated charge $\delta \rho_{\text {inc }} \equiv s^{2} T \delta(\rho / s)$ diffuse with the usual diffusivity $D$ of relativistic hydrodynamics (see, e.g., Ref. [63]). $D$ obeys the Einstein relation $D=\sigma_{\text {inc }}^{\mathrm{dc}} / \chi_{\text {inc }}$ where $\sigma_{\text {inc }}^{\mathrm{dc}}=$ $(s T+\mu \rho)^{2} \sigma_{o}$ and $\chi_{\text {inc }}$ is the static susceptibility of $\delta \rho_{\text {inc }}$. While in general $\chi_{\text {inc }}$ depends in a complicated way on the thermodynamic properties of the state, near a QCP it simplifies to $\chi_{\text {inc }}=\rho^{2} T^{2}(\partial s / \partial T)_{\rho}$ [45]. Furthermore, as $\sigma_{\text {inc }}^{\text {dc }}$ is related to the open-circuit thermal conductivity $\kappa$ by $\sigma_{\text {inc }}^{\mathrm{dc}}=T \rho^{2} \kappa$ in a relativistic hydrodynamic system [45], near the QCP $D$ is equal to the thermal diffusivity $D_{T} \equiv \kappa /(T \partial s / \partial T)_{\rho}$. Using our explicit results [Eq. (13)] for holographic theories, in addition to the temperature scaling of $s$, both diffusivities near the QCP can be written simply as Eq. (3).

The relation [Eq. (3)] is possible because $D_{T}, v_{B}$, and $\tau_{\text {eq }}$ are all related to near-horizon properties of the dual black hole [64]. This fact also lies behind the existence of a relation analogous to Eq. (3) for $z \neq 1$ QCPs, with $\tau_{\text {eq }}$ replaced by $\tau_{P}[25,34]$. But unlike in those cases, where $D_{T}$ and $v_{B}$ are both properties of the QCP, for the $z=1$ cases at hand the relation Eq. (3) relies crucially on the fact that both $D_{T}$ and $\tau_{\text {eq }}$ depend in the same way on the irrelevant deformation away from the QCP sourced by $A_{0}$. This is also different to the case of $z=\infty, \theta=0$ QCPs, where a relation similar to Eq. (3) with $\tau_{\text {eq }}$ replaced by $\tau_{P}$ arises due to the fact that both $D_{T}$ and $v_{B}^{2}$ are determined by the same irrelevant coupling [35-37].

At times $t \lesssim \tau_{\text {eq }}$, relativistic hydrodynamics is not applicable to the system since it doesn't incorporate the dynamics of the slowly relaxing mode that appears at times $t \sim \tau_{\text {eq }}$. Since we expect typical excitations near the QCP to have lifetimes $\sim T^{-1} \ll \tau_{\text {eq }}$, then it may be possible to identify an effective theory valid to earlier times $t \gtrsim T^{-1}$ by supplementing the hydrodynamic equations to incorporate the existence of the slowly relaxing mode [66]. In the Supplemental Material [76], we compute holographically the other entries in the matrix of retarded Green's functions for $J$ and $P$ and show they match those of a hydrodynamic theory with a slowly decaying mode $J_{\text {inc }}: \partial_{t} J_{\text {inc }}=$ $-J_{\text {inc }} / \tau_{\text {eq }}$, using standard techniques $[63,77,78]$. Such effective theories typically display pole collisions in the lower half frequency plane, whereby a diffusive mode acquires a real part and turns into a propagating mode at short distances. The velocity $v_{s}$ of this propagating mode then determines the diffusivity $D=v_{s}^{2} \tau_{\text {eq }}$ (see, eg, (2.17) of Ref. [67]). For Eq. (3) to take this form, we require a velocity $v_{s}^{2}=2 v_{B}^{2} /(d+1-\theta)=1 /(d-\theta)$. It is known $[49,50]$ that $z=1, \theta \neq 0$ theories contain a mode with this velocity in their spectrum. We therefore conjecture that this mode of the IR spacetime is promoted to a mode of the full asymptotically AdS spacetime [79], and thus it is because $D=v_{s}^{2} \tau_{\text {eq }}$ that Eq. (3) is realized. We plan to confirm this picture in Ref. [80], using the techniques developed in Ref. [68]. 
In light of this discussion, it would be interesting to identify for $z \neq 1$ QCPs (where the irrelevant deformation is unimportant) a lifetime $\tau_{\text {eq }} \sim 1 / T$ and velocity $v_{s}^{2} \sim v_{B}^{2}$ of a collective mode such that $D_{T}=v_{s}^{2} \tau_{\text {eq }}$. Such a relation would indicate that it is not the butterfly velocity $v_{B}$ that fundamentally sets the thermal diffusivity, but instead that Eq. (3) arises due to a relation between the velocities of collective modes and the butterfly velocity near quantum critical points.

Breakdown of $\omega / T$ scaling.-The existence of a collective mode with the parametrically long lifetime $\tau_{\mathrm{eq}}$ is the most striking consequence of the breakdown in quantum critical scaling caused by the dangerously irrelevant coupling $A_{0}$, but it is not the only one. It was previously shown that the conductivity $\sigma_{\text {inc }}(\omega, T)$ does not exhibit $\omega / T$ scaling near the QCPs [Eq. (5)]: specifically, $\sigma_{\text {inc }}(\omega$, $T=0) \sim \omega^{-\zeta}[29,30,43]$ while $\sigma_{\text {inc }}^{\text {dc }} \sim T^{\zeta+2(d-\theta)}$ [22]. By carefully keeping track of the dependence on $A_{0}$ [45], we can explicitly attribute this breakdown in $\omega / T$ scaling to the presence of the irrelevant coupling in the IR theory:

$\sigma_{\text {inc }}^{\mathrm{dc}} \sim T^{d-\theta+2 \Delta_{A_{0}}}, \quad \sigma_{\text {inc }}(T=0) \sim A_{0}^{4} \omega^{d-\theta-2 \Delta_{A_{0}}}$.

Recalling that $\Delta_{A_{0}}=(d-\theta+\zeta) / 2$, it is clear that when $z=1$ we can consistently assign $\sigma_{\text {inc }}$ the dimension $\zeta+2(d-\theta)$, and that $\omega / T$ scaling fails because of the nontrivial dependence of $\sigma_{\text {inc }}$ on the irrelevant coupling $A_{0}$.

In contrast, near the $z \neq 1$ counterparts of the QCPs [Eq. (5)] where $A_{0}$ sources a marginal deformation $\Delta_{A_{0}}=0$, the incoherent conductivity obeys $\omega / T$ scaling: $\sigma_{\text {inc }}(\omega, T=0) \sim \omega^{2+(d-2-\theta) / z} \quad[23,29,30,43] \quad$ and $\quad \sigma_{\text {inc }}^{\text {dc }} \sim$ $T^{2+(d-2-\theta) / z}[22]$.

In both cases $(z=1$ and $z \neq 1)$, the scaling theory required to account for the total dimension of $\sigma_{\text {inc }}$ is nontrivial. It involves anomalous dimensions for both the entropy density $\Delta_{s}=d-\theta$ (i.e., hyperscaling violation) and the charge density $\Delta_{\rho}=d-\theta+\Phi[23,43,44]$ and is explained in more detail in Ref. [45]. The anomalous dimension for charge density $\Phi$ is related to the profile of the Maxwell field [Eq. (7)] by $\Phi=(\zeta+\theta-d) / 2$, and thus $\Delta_{\rho}=\Delta_{A_{0}}$ (consistent with our previous observation that $\rho \propto A_{0}$ at $\left.T=0\right)$. Note that the close relation between $\rho$ and $A_{0}$, supplemented by a matched asymptotics argument, is at the root of why the charge response near the QCP is sensitive to the irrelevant deformation sourced by $A_{0}$.

Both the anomalous dimensions, and the extra dimensionful coupling $A_{0}$, permit a much richer family of $T$ dependence in the quantum critical contribution to the conductivity [Eq. (15)] than is allowed in a simple scale invariant theory $[23,44]$, and may be necessary to explain the various scalings observed in strange metals $[46,47]$.

We would like to thank Sean Hartnoll, Jelle Hartong, Elias Kiritsis, and Jan Zaanen for stimulating and insightful discussions. R. A. D. is supported by the Gordon and Betty
Moore Foundation Grant No. GBMF-4306, STFC Ernest Rutherford Grant No. ST/R004455/1 and STFC Consolidated Grant No. ST/P000681/1. The work of S. A. G. was supported by the Delta-Institute for Theoretical Physics (D-ITP) that is funded by the Dutch Ministry of Education, Culture and Science (OCW). B. G. was supported by the European Research Council (ERC) under the European Union Horizon 2020 Research and Innovation Programme (Grant Agreement No. 758759) and was also partially supported during this work by the Marie Curie International Outgoing Fellowship No. 624054 within the 7th European Community Framework Programme FP7/ 2007-2013, as well as by the ERC advanced Grant No. 341222. R. A. D. and B. G. wish to thank Nordita for hospitality during the program "Bounding Transport and Chaos in Condensed Matter and Holography."

*davison@damtp.cam.ac.uk

†s.a.gentle@uu.nl

"blaise.gouteraux@polytechnique.edu

[1] We will set $\hbar=k_{B}=1$ from now on.

[2] S. H. Shenker and D. Stanford, Black holes and the butterfly effect, J. High Energy Phys. 03 (2014) 067.

[3] A. Kitaev, Proceedings at KITP, Proceedings at 'Entanglement in Strongly-Correlated Quantum Matter', KITP (2015).

[4] J. Maldacena, S. H. Shenker, and D. Stanford, A bound on chaos, J. High Energy Phys. 08 (2016) 106.

[5] J. Maldacena and D. Stanford, Remarks on the Sachdev-YeKitaev model, Phys. Rev. D 94, 106002 (2016).

[6] M. P. Heller, Holography, hydrodynamization and heavyion collisions, Acta Phys. Pol. B 47, 2581 (2016).

[7] A. Eberlein, V. Kasper, S. Sachdev, and J. Steinberg, Quantum quench of the Sachdev-Ye-Kitaev Model, Phys. Rev. B 96, 205123 (2017).

[8] M. Gurvitch and A. T. Fiory, Resistivity of $\mathrm{La}_{1.825} \mathrm{Sr}_{0.175} \mathrm{CuO}_{4}$ and $\mathrm{YBa}_{2} \mathrm{Cu}_{3} \mathrm{O}_{7}$ to $1100 \mathrm{k}$ : Absence of Saturation and Its Implications, Phys. Rev. Lett. 59, 1337 (1987).

[9] J. C. Zhang, E. M. Levenson-Falk, B. J. Ramshaw, D. A. Bonn, R. Liang, W. N. Hardy, S. A. Hartnoll, and A. Kapitulnik, Anomalous thermal diffusivity in underdoped $\mathrm{YBa}_{2} \mathrm{Cu}_{3} \mathrm{O}_{6+x}$, Proc. Natl. Acad. Sci. U.S.A. 114, 5378 (2017).

[10] J. Zaanen, Superconductivity: Why the temperature is high, Nature (London) 430, 512 (2004).

[11] J. A. N. Bruin, H. Sakai, R. S. Perry, and A. P. Mackenzie, Similarity of scattering rates in metals showing t-linear resistivity, Science 339, 804 (2013).

[12] S. A. Hartnoll, Theory of universal incoherent metallic transport, Nat. Phys. 11, 54 (2015).

[13] J. Gooth, F. Menges, N. Kumar, V. Süb, C. Shekhar, Y. Sun, U. Drechsler, R. Zierold, C. Felser, and B. Gotsmann, Thermal and electrical signatures of a hydrodynamic electron fluid in tungsten diphosphide, Nat. Commun. 9, 4093 (2018).

[14] J. Zaanen, Planckian dissipation, minimal viscosity and the transport in cuprate strange metals, SciPost Phys. 6, 061 (2019). 
[15] S. Sachdev, Quantum Phase Transitions (Cambridge University Press, Cambridge, England, 2011).

[16] W. Götze and P. Wölfle, Homogeneous dynamical conductivity of simple metals, Phys. Rev. B 6, 1226 (1972).

[17] A. Rosch and N. Andrei, Conductivity of a Clean OneDimensional Wire, Phys. Rev. Lett. 85, 1092 (2000).

[18] S. A. Hartnoll and D. M. Hofman, Locally Critical Resistivities from Umklapp Scattering, Phys. Rev. Lett. 108, 241601 (2012).

[19] S. A. Hartnoll, R. Mahajan, M. Punk, and S. Sachdev, Transport near the Ising-nematic quantum critical point of metals in two dimensions, Phys. Rev. B 89, 155130 (2014).

[20] A. A. Patel and S. Sachdev, DC resistivity at the onset of spin density wave order in two-dimensional metals, Phys. Rev. B 90, 165146 (2014).

[21] S. Sachdev, Quantum Phase Transitions (Cambridge University Press, Cambridge, England, 2011), Chap. 18.

[22] R. A. Davison, B. Goutéraux, and S. A. Hartnoll, Incoherent transport in clean quantum critical metals, J. High Energy Phys. 10 (2015) 112.

[23] B. Goutéraux, Charge transport in holography with momentum dissipation, J. High Energy Phys. 04 (2014) 181.

[24] R. A. Davison and B. Goutéraux, Dissecting holographic conductivities, J. High Energy Phys. 09 (2015) 090.

[25] M. Blake, R. A. Davison, and S. Sachdev, Thermal diffusivity and chaos in metals without quasiparticles, Phys. Rev. D 96, 106008 (2017).

[26] A. Rosch, Interplay of Disorder and Spin Fluctuations in the Resistivity Near a Quantum Critical Point, Phys. Rev. Lett. 82, 4280 (1999).

[27] J. Sirker, R. G. Pereira, and I. Affleck, Diffusion and Ballistic Transport in One-Dimensional Quantum Systems, Phys. Rev. Lett. 103, 216602 (2009).

[28] S. A. Hartnoll, D. M. Hofman, M. A. Metlitski, and S. Sachdev, Quantum critical response at the onset of spin density wave order in two-dimensional metals, Phys. Rev. B 84, 125115 (2011).

[29] C. Charmousis, B. Goutéraux, B. S. Kim, E. Kiritsis, and R. Meyer, Effective holographic theories for low-temperature condensed matter systems, J. High Energy Phys. 11 (2010) 151.

[30] B. Goutéraux and E. Kiritsis, Generalized holographic quantum criticality at finite density, J. High Energy Phys. 12 (2011) 036.

[31] T. Hartman, S. A. Hartnoll, and R. Mahajan, Upper Bound on Diffusivity, Phys. Rev. Lett. 119, 141601 (2017).

[32] A. Lucas, Constraints on hydrodynamics from many-body quantum chaos, arXiv:1710.01005.

[33] M. Blake, Universal Charge Diffusion and the Butterfly Effect in Holographic Theories, Phys. Rev. Lett. 117, 091601 (2016).

[34] M. Blake, Universal diffusion in incoherent black holes, Phys. Rev. D 94, 086014 (2016).

[35] Y. Gu, X.-L. Qi, and D. Stanford, Local criticality, diffusion and chaos in generalized Sachdev-Ye-Kitaev models, J. High Energy Phys. 05 (2017) 125.

[36] M. Blake and A. Donos, Diffusion and Chaos from near $\mathrm{AdS}_{2}$ horizons, J. High Energy Phys. 02 (2017) 013.

[37] R. A. Davison, W. Fu, A. Georges, Y. Gu, K. Jensen, and S. Sachdev, Thermoelectric transport in disordered metals without quasiparticles: The Sachdev-Ye-Kitaev models and holography, Phys. Rev. B 95, 155131 (2017).

[38] A. A. Patel and S. Sachdev, Quantum chaos on a critical Fermi surface, Proc. Natl. Acad. Sci. U.S.A. 114, 1844 (2017).

[39] Y. Werman, S. A. Kivelson, and E. Berg, Quantum chaos in an electron-phonon bad metal, arXiv:1705.07895.

[40] M. Blake, H. Lee, and H. Liu, A quantum hydrodynamical description for scrambling and many-body chaos, J. High Energy Phys. 10 (2018) 127.

[41] Our holographic systems have the minimum allowed value of the Lyapunov time $\tau_{L}=\tau_{P} /(2 \pi),[2,4]$.

[42] M. Blake, R. A. Davison, S. Grozdanov, and H. Liu, Many-body chaos and energy dynamics in holography, J. High Energy Phys. 10 (2018) 035.

[43] B. Goutéraux, Universal scaling properties of extremal cohesive holographic phases, J. High Energy Phys. 01 (2014) 080.

[44] A. Karch, Conductivities for hyperscaling violating geometries, J. High Energy Phys. 06 (2014) 140.

[45] R. A. Davison, S. A. Gentle, and B. Goutéraux, Impact of irrelevant deformations on thermodynamics and transport in holographic quantum critical states, arXiv:1812.11060.

[46] S. A. Hartnoll and A. Karch, Scaling theory of the cuprate strange metals, Phys. Rev. B 91, 155126 (2015).

[47] P. Phillips and C. Chamon, Breakdown of One-Parameter Scaling in Quantum Critical Scenarios for High-Temperature Copper-Oxide Superconductors, Phys. Rev. Lett. 95, 107002 (2005).

[48] L. Huijse, S. Sachdev, and B. Swingle, Hidden Fermi surfaces in compressible states of gauge-gravity duality, Phys. Rev. B 85, 035121 (2012).

[49] I. Kanitscheider and K. Skenderis, Universal hydrodynamics of non-conformal branes, J. High Energy Phys. 04 (2009) 062.

[50] B. Goutéraux, J. Smolic, M. Smolic, K. Skenderis, and M. Taylor, Holography for Einstein-Maxwell-dilaton theories from generalized dimensional reduction, J. High Energy Phys. 01 (2012) 089.

[51] S. A. Hartnoll and L. Huijse, Fractionalization of holographic Fermi surfaces, Classical Quantum Gravity 29, 194001 (2012).

[52] A. Adam, B. Crampton, J. Sonner, and B. Withers, Bosonic fractionalisation transitions, J. High Energy Phys. 01 (2013) 127.

[53] B. Goutéraux and E. Kiritsis, Quantum critical lines in holographic phases with (un)broken symmetry, J. High Energy Phys. 04 (2013) 053.

[54] G. Policastro, D. T. Son, and A. O. Starinets, From AdS/ CFT correspondence to hydrodynamics, J. High Energy Phys. 09 (2002) 043.

[55] P. K. Kovtun and A. O. Starinets, Quasinormal modes and holography, Phys. Rev. D 72, 086009 (2005).

[56] M. Edalati, J. I. Jottar, and R. G. Leigh, Shear modes, criticality and extremal black holes, J. High Energy Phys. 04 (2010) 075.

[57] M. Edalati, J. I. Jottar, and R. G. Leigh, Holography and the sound of criticality, J. High Energy Phys. 10 (2010) 058.

[58] A. Buchel, M. P. Heller, and R. C. Myers, Equilibration Rates in a Strongly Coupled Nonconformal Quark-Gluon Plasma, Phys. Rev. Lett. 114, 251601 (2015). 
[59] R. A. Janik, G. Plewa, H. Soltanpanahi, and M. Spalinski, Linearized nonequilibrium dynamics in nonconformal plasma, Phys. Rev. D 91, 126013 (2015).

[60] A. Buchel and A. Day, Universal relaxation in quark-gluon plasma at strong coupling, Phys. Rev. D 92, 026009 (2015).

[61] W. Sybesma and S. Vandoren, Lifshitz quasinormal modes and relaxation from holography, J. High Energy Phys. 05 (2015) 021.

[62] We restrict to the linear response dynamics around an equilibrium, thermal state.

[63] P. Kovtun, Lectures on hydrodynamic fluctuations in relativistic theories, J. Phys. A 45, 473001 (2012).

[64] The butterfly velocity was computed in terms of the metric near the black hole horizon for the states (5) in Refs. [33,65].

[65] D. A. Roberts and B. Swingle, Lieb-Robinson Bound and the Butterfly Effect in Quantum Field Theories, Phys. Rev. Lett. 117, 091602 (2016).

[66] There are other instances in which holographic theories are known to support anomalously long-lived modes with a variety of interesting dispersion relations (typically involving probe branes or higher-derivative actions [67-75]).

[67] R. A. Davison and B. Goutéraux, Momentum dissipation and effective theories of coherent and incoherent transport, J. High Energy Phys. 01 (2015) 039.

[68] S. Grozdanov, A. Lucas, and N. Poovuttikul, Holography and hydrodynamics with weakly broken symmetries, Phys. Rev. D 99, 086012 (2019).

[69] A. Karch, D. T. Son, and A. O. Starinets, Zero Sound from Holography, Phys. Rev. Lett. 102, 051602 (2009).

[70] S. A. Hartnoll, J. Polchinski, E. Silverstein, and D. Tong, Towards strange metallic holography, J. High Energy Phys. 04 (2010) 120.
[71] R. A. Davison and A. O. Starinets, Holographic zero sound at finite temperature, Phys. Rev. D 85, 026004 (2012).

[72] W. Witczak-Krempa, Quantum critical charge response from higher derivatives in holography, Phys. Rev. B 89, 161114(R) (2014).

[73] S. Grozdanov, N. Kaplis, and A. O. Starinets, From strong to weak coupling in holographic models of thermalization, J. High Energy Phys. 07 (2016) 151.

[74] S. Grozdanov and A. O. Starinets, Second-order transport, quasinormal modes and zero-viscosity limit in the GaussBonnet holographic fluid, J. High Energy Phys. 03 (2017) 166.

[75] C.-F. Chen and A. Lucas, Origin of the drude peak and of zero sound in probe brane holography, Phys. Lett. B 774, 569 (2017).

[76] See Supplemental Material at http://link.aps.org/ supplemental/10.1103/PhysRevLett.123.141601 for more details.

[77] L. P. Kadanoff and P.C. Martin, Hydrodynamic equations and correlation functions, Ann. Phys. (N.Y.) 24, 419 (1963).

[78] S. A. Hartnoll, P. K. Kovtun, M. Muller, and S. Sachdev, Theory of the Nernst effect near quantum phase transitions in condensed matter, and in dyonic black holes, Phys. Rev. B 76, 144502 (2007).

[79] This propagating mode will be in addition to the momentumcarrying sound mode, which has a distinct velocity $\sqrt{\partial p / \partial \epsilon}$.

[80] R. A. Davison, B. Goutéraux, and N. Poovuttikul (to be published).

Correction: The omission of a support statement in the Acknowledgments section has been fixed. 